\title{
Femtosecond Laser-Assisted in Situ Keratomileusis with Topography-Guided or Asphericity-Adjusted Derived Data: A Comparative Contralateral Eye Study
}

Ermano M Alves ( $\nabla$ ermanomelo@gmail.com )

Oftalmax

Adriana F Lyra

Hospital Santa Luzia

Manuela Tenório

Oftalmax

Natália Mesquita

Oftalmax

Carolina Bacelar

Hospital Santa Luzia

Afra Montenegro

Oftalmax

Lucas Alves

FAV

Márcio Alves

FPS-IMIP

\section{Research Article}

Keywords: femtosecond LASIK, femtoLASIK, topography-guided LASIK, asphericity-guided LASIK, T-CAT, Custom-Q, Contoura

Posted Date: November 10th, 2021

DOI: https://doi.org/10.21203/rs.3.rs-1058148/v1

License: (c) (i) This work is licensed under a Creative Commons Attribution 4.0 International License.

Read Full License

Version of Record: A version of this preprint was published at BMC Ophthalmology on April 25th, 2022. See the published version at https://doi.org/10.1186/s12886-022-02407-w. 


\section{Abstract}

Background: Wavefront-optimized laser-assisted in situ keratomileusis (LASIK) ablation is the most commonly performed procedure in refractive surgery, but new technologies have become available. Our goal was to compare topography-guided (Contoura) and asphericity-guided (Custom-Q) customized ablation treatments for the correction of myopia with or without astigmatism.

Methods: This prospective, randomized, double-blind, contralateral eye study included 60 eyes of 30 patients with myopia or myopic astigmatism requiring femtosecond LASIK (FemtoLASIK) treatment. For each patient, one eye was randomized to undergo Contoura treatment, and the other underwent Custom-Q abaltion. Uncorrected distance visual acuity (UDVA), corrected distance visual acuity (CDVA), manifest refractive spherical equivalent (MRSE), sphere (SPH), cylinder (CYL), 6.0-mm total corneal aberration root mean square (RMS), coma (COMA), trefoil (TREF), and spherical aberration (SA) were measured and analysed after a 1-year follow-up.

Results: The UDVA was $-0.08 \pm 0.06 \log M A R$ in Contoura eyes and $-0.08 \pm 0.05$ logMAR in Custom-Q eyes $(p=0.309)$ after 12 months. Twenty-five eyes (83\%) in the Contoura group and twenty-six eyes (87\%) in the Custom-Q group had a UDVA of $20 / 16$ at the end of 12 months, and $100 \%$ of eyes in both groups reached a UDVA of $20 / 25$ or better. Ninety and $100 \%$ of eyes in the Contoura and Custom-Q groups, respectively, achieved a residual $C Y L \leq 0.50 \mathrm{D}(\mathrm{p}=0.237)$. No statistically significant difference was observed between the surgical techniques in the preoperative to 1-year postoperative changes for any of the parameters evaluated (MRSE, CYL, RMS, DEF, COMA, TREF, and SA).

Conclusions: The Contoura and Custom-Q techniques yielded excellent visual and refractive results, but the evidence did not reveal any clear differences between these two methods after one year of follow-up.

Trial registration: RBR-8rs5kt Myopia and Astigmatism Topography-guided Refractive Surgery by Contoura Method Versus Customized by Asphericity in Contralateral Eyes: A prospective Double-blind Randomized Study. UTN code: U111-1243-7257

\section{Background}

The prevalence of myopia is increasing; approximately $50 \%$ of the world population is estimated to present with this condition by 2050 [1]. At the same time, the evolution of refractive surgery towards higher levels of safety and satisfaction has led to the development of different treatment techniques and ablation profiles with excimer lasers [2, 3]. Customized treatments of refractive errors have been available for many years, involving wavefront-, topography-, asphericity-, or ablation-guided optimizations that have achieved outcomes superior to those observed with conventional ablation [4-8].

Typically, the human cornea is aspherical and prolate (i.e., it is more curved at the centre and flatter at the periphery). However, conventional refractive surgeries for myopia correction induce changes in corneal asphericity, primarily by turning the prolate shape of the corneal surface into an oblate shape (i.e., flatter 
at the centre and more curved at the periphery). These changes may increase ocular aberrations, specifically spherical aberrations, which result in poor postoperative visual acuity in low-light conditions [8].

Physiological corneal asphericity varies significantly across individuals and can be measured by using a concept called the $\mathrm{Q}$ factor [9]; a $\mathrm{Q}$ factor of -1 to 0 indicates a prolate corneal surface, while a $\mathrm{Q}$ factor $>0$ indicates and oblate surface, and a spherical shape is represented by a $Q$ factor of 0 [9]. Normal corneas usually have a minimal $Q$ value ranging from -0.23 to -0.30 and a positive spherical aberration (SA) of approximately $+0.27 \mu \mathrm{m}$ [10]. The Q-factor customized corneal ablation aims to correct refractive errors by modifying as little as possible the preoperative $\mathrm{Q}$ and $\mathrm{SA}$.

Approximately $90 \%$ of optical aberrations of the human eye are derived from corneal surface irregularities [11]. Over the years, the application of topography-guided excimer laser ablation in irregular corneas, decentred or small optic zones after refractive surgery and even in ectatic corneas has been optimized with excellent results [12-15]. Several studies have suggested using this technique in normal corneas, and the results have been shown to be superior to those obtained with currently used ablation techniques $[4-6,8]$.

In 2013, the U.S. Food and Drug Administration (FDA) approved the use of WaveLight ${ }^{\circledR}$ topographyguided customized ablation treatment (T-CAT) [16]in the United States for treating myopia in regular corneas with or without astigmatism. This type of treatment aims to correct small irregularities in normal corneas that are responsible for high-order optical aberrations (HOAs), which, at least in principle, influence the final clinical refraction. Following correction of these small changes by the topographyguided laser, the final refraction to be treated must also experience small changes. This approach would thus lead to better visual acuity and fewer optical aberrations with respect to other techniques [10].

Potential modifications in topography-based refraction for achieving a visual acuity greater than 20/20 and better visual quality, however, require further investigation, and to date, there is no consensus on the criteria for modifying the final treatment and whether this approach is superior to other ablation profiles already widely used by refractive surgeons.

In this study, we aimed to compare two methods of corneal ablation performed using femtosecond laserassisted in situ keratomileusis (LASIK) in myopic eyes with or without astigmatism in virgin eyes. For each patient, one eye was treated with T-CAT, and the contralateral eye was treated with Custom-Q. Both methods required the use of WaveLight software.

\section{Methods}

\section{Study design}

This prospective, randomized, double-blind, comparative study was conducted at the OFTALMAX Eye Clinic and Surgery in Recife, Pernambuco, Brazil. Fifty-one patients were selected between August and 
December 2018 at the Santa Luzia Foundation in Recife, an ophthalmological care facility affiliated with the Unified Health Service in Brazil. The study was approved by the ethics committee of the Hospital Complex HUOC/PROCAPE after informed consent was and was conducted following the guidelines of the Declaration of Helsinki. The study included patients aged 20 to 35 years of both sexes with myopia with or without astigmatism, with a stable refractive error ranging between -0.50 and -8.00 spherical dioptres (D) and astigmatism between 0.00 and -3.00 cylindrical dioptres (CD) for a minimum of 1 year, a maximum spherical equivalent of $8.00 \mathrm{D}$, and a corrected visual acuity of $0.1 \log \mathrm{MAR}$ or better. The exclusion criteria were any clinical condition that could modify the surgical outcome, such as anisometropia with a refractive error of more than $1.00 \mathrm{D}$ in spherical or $0.75 \mathrm{D}$ in astigmatism, clinical signs of dry eye, cataracts, corneal scarring, or neovascularization within $1.0 \mathrm{~mm}$ of the intended ablation zone; epithelial basement membrane dystrophy; history of recurrent corneal erosion; pachymetry below $500 \mu \mathrm{m}$; suspicion of subclinical or established keratoconus; macular or retinal disease; diagnosis of glaucoma or ocular hypertension; current use of systemic corticosteroids or immunosuppressive therapy; collagen diseases; vascular diseases; diabetes mellitus types I and II; pregnancy and breastfeeding.

\section{Preoperative examinations}

Preoperative examinations included measurement of visual acuity using a digital projector (Apramed CB 300 ) without distance correction and then with static refraction after 30 min using $1 \%$ cyclopentolate hydrochloride (Allergan); measurement of intraocular pressure using a Goldmann tonometer; anterior segment biomicroscopy; retinal mapping by indirect ophthalmoscopy; complete anterior segment analysis using dual Scheimpflug-based corneal system tomography (Galilei Ziemer Ophthalmic Systems AG, Port, Switzerland); measurement of asphericity and analysis of corneal HOAs using Wavelight Topolyzer Vario software (Alcon Laboratories, Inc., Fort Worth, Texas) and acquisition of a minimum of eight, good-quality, reproducible images. The images were digitally transferred to an EX500 (Alcon) Excimer Laser workstation and used to plan the ablation profile. Patients were asked to discontinue wearing contact lenses, if applicable, 1 week before the screening (soft contact lenses) or 4 weeks before screening (rigid gas-permeable contact lenses).

\section{Surgeries}

The surgeries were performed on two consecutive days and involved the entire team of investigators and two Alcon WaveLight consultants. All preoperative examination outcomes were assessed, and images were acquired on the day of the surgery via Topolyzer Vario (WaveLight) software. All patients were scheduled for simultaneous FemtoLASIK in both eyes. Visual correction was performed according to the cycloplegic refraction with T-CAT using the commercially called Contoura method in group 1 and Custom$\mathrm{Q}$ in group 2 (contralateral eye); final goal was to achieve emmetropia in all patients.

Randomization was ensured using a spreadsheet created in Excel. Consequently, following the order of the patients admitted to the block, even-numbered patients were administered Contoura treatment in the right eye and Custom-Q in the left eye, while the odd-numbered patients were treated with Contoura in their left eye and Custom- $\mathrm{Q}$ in the right eye. 
Contoura surgical planning was performed following the guidelines updated by Alcon technical engineers according to the following steps:

1. Acquisition of a minimum of eight good-quality topographic images using Topolyzer Vario software.

2. Comparison between the pupillary and mid-peripheral areas using the Compare Images Display and identification of four or more images with a refractive error of less than $0.75 \mathrm{D}$ in the treatment area demonstrating a good definition of the limbus and pupillary margin.

3. Transfer of images via a USB stick to the EX500 (Alcon) Excimer Laser workstation. Images with a value of 3 were considered high-quality (i.e., good quality for eventual cyclotorsion control) using the Quality Dynamic Link Library.

4. Exclusion of lower-quality images with a refractive error $\geq 0.75 \mathrm{D}$ in the treatment area until a minimum of four images were obtained with a mean average deviation (MAD) of the axis of astigmatism of 0.5 .

5. Import of static refraction data into Contoura planning software;

6. Zeroing of astigmatism and spherical degree to visualize the ablation pattern of the HOAs;

7. Determination of the amount of ablated tissue in the central and peripheral regions of the cornea. The difference was not permitted to exceed 3 microns; if this occurred, the spherical correction was altered until this limit was achieved.

8) Observation of the difference between the topographic cylinder measured using Topolyzer Vario software and the manifest refractive cylinder, with final treatment corrected according to the following parameters:

a- When the refractive cylinder was smaller than the topographic cylinder, the average between the two measurements was obtained, and treatment was performed with the final mean value while maintaining the topographic axis.

b- When the refractive cylinder was larger than the topographic cylinder, treatment was performed with the axis and the total cylinder of the topographic cylinder.

9) Computation of the spherical degree requiring correction after adjusting the spherical equivalent of the change in astigmatism power. This value could vary according to the WaveLight correction table.

Treatment using Custom-Q was performed using the intended asphericity, which was identical to that of the initial asphericity measured using Topolyzer Vario software; furthermore, the HOAs of the cornea were left uncorrected. Both treatments were centred on the corneal vertex.

Safety was ensured by measuring the percentage of altered tissue (PTA) using the corneal thickness at its thinnest region when calculating the ablation $[17,18]$. All surgeries were performed by an experienced surgeon (EMA) at the OFTALMAX operating room using a WaveLight@ EX500 (Alcon) Excimer Laser according to data provided by Topolyzer Vario software (WaveLight). A corneal flap was created using a 
femtosecond Z8 laser (Ziemer Ophthalmic Systems, Switzerland). The flap thickness was 110 microns, the flap diameter was $9.0-9.5 \mathrm{~mm}$, and the optical zone was $6.0-6.5 \mathrm{~mm}$ in all treatments.

\section{Postoperative follow-up}

In the immediate postoperative period, topical treatment was initiated with a combination of moxifloxacin and dexamethasone (Vigadexa, Alcon) four times a day for 1 week, and lubricant eye drops containing sodium hyaluronate and carboxymethylcellulose (Optive, Allergan) were provided by Oftalmax. The patients were examined by medical researchers who did not participate in the randomization at day 1 , week 1, month 1, 3 months and 1 year after the procedure.

During the first three postoperative follow-up visits, possible complications and ocular surface integrity were assessed by anterior segment biomicroscopy, uncorrected distance visual acuity (UDVA), and fundoscopy. The last two visits included the evaluation of the patients' visual acuity with cycloplegic refraction, retinal mapping, applanation tonometry, and total anterior segment analysis performed by dual Scheimpflug-based corneal system tomography.

Among the 51 patients initially selected for the study, 13 were excluded. After PTA analysis, five patients did not appear to be good candidates for flap creation and underwent photorefractive keratectomy; one presented with a flap complication (the vertical cut for the flap margin was not performed), and a second flap was required after $1 \mathrm{~h}$ in the same eye; four did not have reliable images produced according to the T-

CAT criteria; one mistakenly underwent bilateral T-CAT ablation; one was purposely undercorrected in both eyes to avoid exceeding the 40\% PTA limit; and one developed severe dry eye keratitis in both eyes, preventing reliable data collection. The final sample included a total of 38 participants, but among them, 8 did not participate in the 1-year final evaluation.

\section{Statistical methodology}

Quantitative data are expressed as the mean, standard deviation, median, minimum, and maximum. Qualitative or categorical variables are described as absolute ( $n$ ) and relative (\%) frequencies and were compared using the chi-square test or Fisher's exact test, in the case of $2 \times 2$ tables, with expected frequencies $<5$.

The Kolmogorov-Smirnov test was used to assess whether the quantitative data had a normal distribution. Student's t-test was used to compare the two surgical techniques for data considered normally distributed, and when normality could not be assumed, the nonparametric Mann-Whitney U test was used. The paired t-test was used to assess differences between pre- and postoperative parameters under the assumption of normality, and the nonparametric Wilcoxon test was used in case the data were not normally distributed.

Regarding the assessment of uncorrected distance visual acuity (UDVA) measured at day 1 (D1), week 1 (W1), 3 months (3M), and 1 year (1Y), a mixed ANOVA model was used for comparing surgical techniques and measurements over time and assessing the interaction effect between the factors 
"surgery" and "time". Regarding the statistical significance of variation over time, the Bonferroni method for multiple comparisons was used for comparisons with the baseline value at day 1 of the surgery.

All analyses were performed using Minitab 18.0 software. $p$ values $<0.05$ were considered statistically significant.

\section{Results}

\section{Demographic data}

A total of 30 patients and 60 surgeries were evaluated ( 30 in the right eye and 30 in the left eye). Patients were between 19 and 33 years old, with a mean age of 26.3 years and standard deviation of 4.3 years. Just over half of the patients were female (56.7\%) (Table 1). The sequence of surgeries in the right and left eyes was not balanced, as the Contoura technique was mostly used on the right eye, while the Custom-Q technique was mostly used on the left eye (73.3\%) (Table 2).

Table 1

Demographic data

\begin{tabular}{|ll|}
\hline Demographic data & Total \\
\hline No. of patients & 30 \\
\hline No. of eyes & 60 \\
\hline Age, years & 30 \\
\hline N & $26.3(4.3)$ \\
\hline mean (sd) & 26 \\
\hline median & $19-33$ \\
\hline min-max & \\
\hline Sex, N (\%) & $17(56.7 \%)$ \\
\hline women & $13(43.3 \%)$ \\
\hline men & \\
\hline sd = standard deviation; min = minimum observed value; max $=$ maximum observed value; \\
\hline
\end{tabular}


Table 2

Operated eye versus surgical technique used

\begin{tabular}{|c|c|c|c|}
\hline & Contoura $(n=30)$ & $\begin{array}{l}\text { Custom-Q } \\
(n=30)\end{array}$ & $\begin{array}{l}\text { Total } \\
(n=60)\end{array}$ \\
\hline right eye, n (\%) & $22(73.3 \%)$ & $8(26.7 \%)$ & $30(50 \%)$ \\
\hline left eye, n (\%) & $8(26.7 \%)$ & $22(73.3 \%)$ & $30(50 \%)$ \\
\hline total, n (\%) & $30(100 \%)$ & $30(100 \%)$ & $60(100 \%)$ \\
\hline
\end{tabular}

No statistically significant difference was found when comparing the Contoura and Custom-Q groups in the parameters measured in the preoperative period (CDVA, spherical equivalent, sphere, cylinder, Sim K, pachymetry), nor in the PTA measured during surgery (Table 3). 
Table 3

Comparison of techniques with regard to the parameters evaluated in the pre- and intraoperative periods

$\begin{array}{ll}\text { Contoura } & \text { Custom- } Q \\ (n=30) & (n=30)\end{array}$

p-value

CDVA (logMAR)

$\begin{array}{llll}\text { mean } \pm \text { sd } & -0.06 \pm 0.06 & -0.06 \pm 0.05 & \\ \text { median } & -0.1 & -0.1 & 0.679^{1} \\ \text { min-max } & -0.1-0.1 & -0.1-0 & \end{array}$

Spher. Equiv. (D)

\begin{tabular}{llll} 
mean \pm sd & $-3.39 \pm 1.65$ & $-3.47 \pm 1.69$ & $0.813^{1}$ \\
median & -2.88 & -2.94 & \\
min-max & $-7.75--1.63$ & $-7.88--1.00$ & $0.678^{1}$ \\
Sphere (D) & & & \\
mean \pm sd & $-2.88 \pm 1.53$ & $-3,03 \pm 1.60$ & \\
median & -2.50 & -2.50 & $0.527^{1}$ \\
min-max & $-7.25--1.25$ & $-7.00--1.00$ & \\
Cylinder (D) & & & \\
mean \pm sd & $-1.02 \pm 0.83$ & $-0.88 \pm 0.71$ & \\
median & -0.88 & -0.75 & \\
min-max & $-3.25-0.00$ & $-2.50-0.00$ & \\
Sim K (D) & & & \\
mean \pm sd & $43.92 \pm 1.49$ & $43.99 \pm 1.48$ & \\
median & 43.89 & 43.95 & \\
min-max & $40.00-48.02$ & $40.36-47.70$ & \\
Pachy (D) & & $544.57 \pm 29.20$ & \\
mean \pm sd & $543.7 \pm 28.30$ & & \\
\hline
\end{tabular}

sd = standard deviation; $\min =$ minimum observed value; $\max =$ maximum observed value; $\mathrm{D}=$ dioptre;

${ }^{1}$ Mann-Whitney test; ${ }^{2}$ Student t-test; 


\begin{tabular}{|c|c|c|c|}
\hline & $\begin{array}{l}\text { Contoura } \\
(n=30)\end{array}$ & $\begin{array}{l}\text { Custom-Q } \\
(\mathrm{n}=30)\end{array}$ & p-value \\
\hline median & 542.00 & 542.00 & $0.872^{2}$ \\
\hline $\min -\max$ & $493.00-600.00$ & $494.00-607.00$ & \\
\hline \multicolumn{4}{|l|}{ PTA (\%) } \\
\hline mean $\pm s d$ & $30.97 \pm 3.13$ & $30.12 \pm 3.12$ & \\
\hline median & 30.10 & 30.11 & $0.322^{1}$ \\
\hline $\min -\max$ & $25.20-39.00$ & $23.80-37.20$ & \\
\hline \multicolumn{4}{|c|}{$\begin{array}{l}\text { sd = standard deviation; } \min =\text { minimum observed value; } \max =\text { maximum observed value; } \mathrm{D}= \\
\text { dioptre; }\end{array}$} \\
\hline \multicolumn{4}{|c|}{${ }^{1}$ Mann-Whitney test; ${ }^{2}$ Student t-test; } \\
\hline
\end{tabular}

\section{Evolution of parameters between the preoperative evaluation and 1-year follow-up}

Both surgical techniques achieved significant increases in the value of parameters, such as the root mean square (RMS), defocus (DEF) and coma (COMA). The SA parameter also presented significant variation between the pre- and postoperative assessments, with a median of $-0.18 \mathrm{D}$ in the preoperative assessment and $-0.27 \mathrm{D}$ and $-0.29 \mathrm{D}$ in the postoperative assessment in the Contoura and Custom-Q groups, respectively.

Only the trefoil (TREF) parameter presented with no significant variation in value in the 1-year follow-up relative to the preoperative period, with $p=0.198$ (Contoura) and $p=0.972$ (Custom-Q) (Table 4). 
Table 4

Comparison of pre- and postoperative values according to the type of surgery performed Contoura $(n=30)$

Custom- $\mathrm{Q}(\mathrm{n}=30)$

pre post p-value pre post p-value

RMS $(\mu \mathrm{m})$
mean \pm
$1.07 \pm 0.45$
$1.67 \pm 0.53$
$0.99 \pm 0.46$
$1.76 \pm 0.59$

sd

median

0.93

1.63

$<0.001^{1}$

0.92

1.61 $<0.001^{1}$

$\min -\max$

$0.49-2.43$

$0.77-3.14$

$0.33-2.19$

$0.74-3.25$

DEF (D)

$\begin{array}{lllllll}\begin{array}{l}\text { mean } \pm \\ \text { sd }\end{array} & 0.28 \pm 0.23 & 1.07 \pm 0.40 & & 0.24 \pm 0.23 & 1.09 \pm 0.43 & \\ \text { median } & 0.32 & 1.06 & <0.001^{2} & 0.29 & 1.06 & <0.001^{2} \\ \text { min-max } & -0.15-0.66 & 0.31-2.16 & & -0.29-0.69 & 0.34-2.11 & \end{array}$

COMA (D)

$\begin{array}{lllll}\text { mean } \pm & 0.18 \pm 0.09 & 0.26 \pm 0.15 & 0.17 \pm 0.08 & 0.29 \pm 0.14\end{array}$

sd

$\begin{array}{lllllll}\text { median } & 0.19 & 0.24 & 0.017^{2} & 0.17 & 0.25 & <0.001^{2} \\ \text { min-max } & 0.02-0.47 & 0.05-0,81 & & 0.04-0.31 & 0.07-0.64 & \end{array}$

TREF (D)
mean \pm
$0.13 \pm 0.08$
$0.13 \pm 0.08$
$0.13 \pm 0.08$
$0.15 \pm 0.07$
sd

median

0.13

0.11

$0.198^{1}$

0.12

0.13

$0.972^{1}$

$\min -\max$

$0.03-0.41$

$0.01-0.31$

$0.02-0.36$

$0.03-0.37$

SA (D)

mean \pm

$-0.17 \pm 0.04 \quad-0.28 \pm 0.09$

$-0.17 \pm 0.05 \quad-0.32 \pm 0.12$

sd

median

$-0.18$

$-0.27$

$<0.001^{2}$

$-0.18$

$-0.29$ $<0.001^{2}$

$\min -\max \quad-0.24--0.05 \quad-0.51--0.06$

$-0.28--0.03 \quad-0.65--0.13$

$\mathrm{sd}=$ standard deviation; $\min =$ minimum observed value; $\max =$ maximum observed value; $\mathrm{D}=$ dioptre;

${ }^{1}$ t-paired test; ${ }^{2}$ Wilcoxon test; 


\section{Comparison of surgical techniques between pre- and postoperative assessments (1-year follow-up)}

No statistically significant differences were observed between the surgical techniques in terms of the preoperative to 1-year postoperative changes for the five parameters evaluated at the 1-year follow-up (RMS, DEF, COMA, TREF, and SA) (Table 5). 
Table 5

Comparison of the preoperative to 1-year postoperative changes in parameters between the types of surgery

\section{Difference between pre-and postoperative value (1 year)}

Contoura Custom-Q p-value

$\operatorname{RMS}(\mu \mathrm{m})$

$\begin{array}{llll}\text { mean } \pm \text { sd } & 0.59 \pm 0.68 & 0.77 \pm 0.67 & \\ \text { median } & 0.58 & 0,82 & 0.311^{1} \\ \text { min-max } & -0.81-2.32 & -0.61-1.96 & \end{array}$

DEF (D)

$\begin{array}{llll}\text { mean } \pm \text { sd } & 0.79 \pm 0.34 & 0.85 \pm 0.40 & \\ \text { median } & 0.71 & 0.78 & 0.712^{2}\end{array}$

$\min -\max \quad 0.31-1.67 \quad 0.26-2.16$

$\operatorname{COMA}(\mathrm{D})$

$\begin{array}{lll}\text { mean } \pm \text { sd } & 0.07 \pm 0.16 & 0.12 \pm 0.14\end{array}$

$\begin{array}{llll}\text { median } & 0.07 & 0.10 & 0.221^{1}\end{array}$

$\min -\max \quad-0.22-0.56 \quad-0.11-0.44$

TREF (D)

$\begin{array}{llll}\text { mean } \pm \mathrm{sd} & -0.001 \pm 0.10 & 0.02 \pm 0.08 & \\ \text { median } & -0.01 & 0.01 & 0.402^{1}\end{array}$

$\begin{array}{lll}\min -\max & -0.19-0.22 \quad-0.24-0.19\end{array}$

SA (D)

mean $\pm s d \quad-0.10 \pm 0.10 \quad-0.14 \pm 0.13$

$\begin{array}{llll}\text { median } & -0.09 & -0.10 & 0.294^{2}\end{array}$

$\min -\max \quad-0.43-0.06 \quad-0.50-0.00$

sd = standard deviation; $\min =$ minimum observed value; $\max =$ maximum observed value; $\mathrm{D}=$ dioptre;

${ }^{1}$ Student t-test; ${ }^{2}$ Mann-Whitney test; 
No interaction was identified between surgical techniques and assessments $(p=0.727)$, indicating that the curves representing the surgeries showed a similar behaviour over time (Figure 1).

Thus, the analysis of the UDVA data showed that regardless of the assessment performed, there were no significant differences between the surgical techniques $(p=0.309)$, but a significant improvement between the assessments at $W 1,3 M$, and $1 Y$ relative to $D 1(p<0.001)$ was identified for both groups (Table 6).

Table 6

Uncorrected distance visual acuity (logMAR)

\begin{tabular}{|lll|}
\hline UDVA & Contoura & Custom-Q \\
\hline Day 1 (baseline) & & \\
\hline mean \pm sd & $-0.01 \pm 0.12$ & $-0.03 \pm 0.11$ \\
\hline median & -0.05 & -0.10 \\
\hline min-max & $-0.10-0.40$ & $-0.12-0.40$ \\
\hline Week 1 & & \\
\hline mean \pm sd & $-0.03 \pm 0.07$ & $-0.06 \pm 0.06$ \\
\hline median & 0.00 & -0.10 \\
\hline min-max & $-0.10-0.18$ & $-0.10-0.10$ \\
\hline 3 months & & \\
\hline mean \pm sd & $-0.07 \pm 0.07$ & $-0.08 \pm 0.05$ \\
\hline median & -0.10 & -0.10 \\
\hline min-max & $-0.30-0.10$ & $-0.10-0.10$ \\
\hline 1 year & & \\
\hline mean \pm sd & $-0.08 \pm 0.06$ & $-0.08 \pm 0.05$ \\
\hline median & -0.10 & -0.10 \\
\hline min-max & $-0.10-0.10$ & $-0.10-0.10$ \\
\hline ANovA mixed model: & \\
\hline
\end{tabular}

comparison of surgical techniques: $p=0.309$

comparison of assessments: $p<0.001$ (multiple comparisons with baseline)

interaction of factors: $p=0.727$ 


\section{Analysis of data from standardized tables: statistical comparison of the Contoura and Custom-Q surgical techniques}

\section{Efficacy}

Twenty-five eyes (83\%) in the Contoura group and twenty-six eyes (87\%) in the Custom-Q group had a UDVA of $20 / 16$ at the end of 12 months, and $100 \%$ in both groups reached a UDVA of 20/25 or better. There was no difference between the groups in terms of the UDVA $(20 / 16$ versus $20 / 20$ and $20 / 25 ; p=$ 1.000 , Fisher's exact test) or preoperative CDVA (20/16 versus $20 / 20$ and $20 / 25 ; p=0.592$, chi-square test) (Figure 2, A and B).

\section{Postoperative UDVA vs. preoperative CDVA}

Only one eye in each group (3\%) experienced loss of one line of sight between preoperative CDVA and postoperative UDVA. There was gain of one line of sight or more in five eyes $(17 \%)$ in the Contoura group and nine eyes (30\%) in the Custom-Q group, but this difference was not statistically significant $(p=0.243$, chi-square test) (Figure 2, C and D).

\section{Safety}

The different ablation profiles had similar safety profiles. There was no change in the CDVA in $77 \%$ of eyes subjected to Contoura or in $63 \%$ in the Custom-Q group ( $p=0.260$ ); furthermore, $23 \%$ and $33 \%$ of the eyes in the Contoura and Custom-Q groups, respectively, gained a line of CDVA $(p=0.390)$ (Figure $3, A$ and B).

\section{Accuracy}

There was no significant difference between the groups in the percentage of eyes that achieved $\pm 0.50 \mathrm{D}$ in the final spherical equivalent (Contoura $80 \%$ vs Custom-Q 90\%; $p=0.278$ ) (Figure 3, C and D).

\section{Spherical equivalent attempted versus achieved}

The scatter plots show that the relationship between the attempted and achieved spherical equivalent correction in both the Contoura and Custom-Q groups at the 1-year follow-up was similar (Figure 4, A and B).

\section{Astigmatism}

Clinical astigmatism was corrected very effectively in both groups, with $100 \%$ of the eyes having a final result of $\leq 1 \mathrm{D}$. Furthermore, $90 \%$ of the eyes in the Contoura group and $100 \%$ of the eyes in the Custom$\mathrm{Q}$ group achieved a residual cylinder of $\leq 0.50 \mathrm{D}(\mathrm{p}=0.237)$ (Figure $4, \mathrm{C}$ and $\mathrm{D})$. 


\section{Discussion}

The main objective of our study was to detect differences in the effectiveness of two surgical techniques (Contoura and Custom-Q) in the correction of myopia with or without astigmatism. Our results indicate that at the 1-year follow-up, the two ablation profiles were shown to be effective and safe, with similar visual and refractive results in all analysis criteria and no significant disagreements between groups.

Among the parameters analysed, the UDVA is the most relevant in refractive surgery, as it reflects the patient's primary objective: achieving good vision without glasses. In our sample, both techniques produced excellent final visual acuity: the Contoura group had a UDVA of 20/20 and 20/16 or better in $93 \%$ and $83 \%$ of eyes, while the Custom-Q group had a UDVA of $20 / 20$ and $20 / 16$ in $97 \%$ and $87 \%$ of eyes, respectively. In both groups, there was a significant improvement in visual acuity over 12 months.

The induction of high-order aberrations, despite being slightly higher in the Custom-Q group, was not significantly different between groups in the final analysis, contradicting the idea of the advantage of topography-guided surgery for this purpose. Regarding efficacy, safety and accuracy, it was also not possible to detect differences between the methods.

Custom-Q ablation attempts to maintain the original asphericity of the cornea, which is directly related to postoperative spherical aberration. Previous studies have shown that the Custom- $Q$ technique causes a smaller change in the Q-value than traditional optimized ablation methods, with a consequent lower induction of spherical aberration $[7,8,19]$. Koller et al. [8] emphasized the potential of custom aspheric ablation to replace optimized ablation and demonstrated that asphericity was less affected in myopia up to $-5 \mathrm{D}$ when using custom ablation than when using wavefront-optimized ablation (WFO). In a retrospective study of consecutive patients, Stojanovic et al. [19] reported good results using Custom-Q and WFO but a lower induction of spherical aberration using Custom-Q. Tawfik et al. [7] similarly suggested replacing WFO with Custom-Q. However, despite these good results and the simplicity of its programming and application, Custom-Q has not gained popularity among refractive surgeons; the good results and ease of use of WFO can be a deterrent when seeking supposedly superior methods.

Contoura topography-guided treatment, on the other hand, modifies the treatment according to the combination of two elements, the clinical and topographic cylinders. This approach has been in use for many years. In 2007, Alpins et al. [20] recommended observing the difference between cylinders to modify the treatment; Kanellopoulus [21] later developed a new surgical planning method called topography-modified refraction (TMR) based on the same principle. Furthermore, Stefano et al. [22] analysed different strategies for correcting astigmatism and found that the UDVA was better than the CDVA in $25 \%$ of eyes treated with T-CAT. Shetty et al. [23] examined 60 eyes of 30 patients and found that the UDVA was $20 / 20$ in $97 \%$ and $93 \%$ of patients in the T-CAT group and the WFO group, respectively, with a lower induction of low- and high-order aberrations in the group. Tiwari et al. [24] evaluated 200 eyes from 100 patients and observed that the UDVA was $20 / 20$ in $90 \%$ and $92 \%$ of patients in the WFO and TCAT groups, respectively, with similar aberration induction in both groups. Kim et al. [25] found that the visual and refractive results were similar in contralateral eyes in the T-CAT and WFO groups; however, 
significantly fewer HOAs were induced with T-CAT. In a retrospective study involving 1274 eyes, Wallerstein et al. [26] highlighted the difference in the axis between clinical and topographic astigmatism. Furthermore, the results of topography-guided surgery were worse when the displacement of the astigmatism axis ranged from 21 to $45^{\circ}$. Ozulken et al. [27] found excellent results using T-CAT over WFO in 32 patients, with less induction of horizontal and vertical coma-type aberrations, a smaller amount of ablated tissue and 20/20 UDVA in 97\% of patients. On the other hand, Zhang et al. [28] reported unfavourable results using topography-guided surgery in 432 virgin eyes of 216 patients, with less accuracy in corrected astigmatism than WFO despite demonstrating less induction of high-order aberrations. Skanchy [29] compared FDA data from three new refractive surgery platforms, namely, Wavefront-guided Visx iDesign, Wavelight TCAT (Contoura) and topography-guided Nidek EC-5000 CaTz, and found excellent results across all platforms; however, the UDVA was significantly better with T-CAT than with the other two platforms. The lack of standardization for the T-CAT is a significant limitation that prevents a comparison of results with an acceptable degree of reliability, as small differences in surgical programming between these different techniques are rarely described in detail in the literature. For this reason, we provided detailed information about the surgical schedule in the T-CAT group (Contoura) in our clinical study.

To the best of our knowledge, this is the first prospective, randomized, double-blind comparative study comparing Contoura and Custom- $\mathrm{Q}$ ablation treatments in normal contralateral eyes. The importance of these results is reinforced by two factors. First, because the study compares both eyes, the patients serve as their own controls, with small differences in refraction and good preoperative corrected visual acuity in both eyes. Second, treatment randomization, surgical planning, surgery, and postoperative evaluations were all performed by different investigators, which significantly increased the likelihood that any differences observed were due to the selected ablation method and not bias.

Nevertheless, this study has several limitations. First, it was not possible to assess contrast sensitivity, which could provide additional information about visual quality and perhaps reveal differences between the two techniques. Second, the sample size was not sufficiently large to show the real advantages of one group over another. Furthermore, the similar results obtained for the two surgical methods may be due to their shared technical characteristics, including centring of the ablation on the apex of the cornea (instead of the pupillary axis) and attempting to maintain the initial asphericity measured by the Topolyzer Vario software. Therefore, at least in principle, the two methods may have had a similar effect on the reduced induction of spherical aberrations. These two factors (apex centring and low aspherical shift) may be more critical in determining postoperative outcomes than the correction of small HOAs in naive eyes or the slight modification of the magnitude and axis of astigmatism. However, more studies are needed to support this hypothesis.

Despite these limitations, it is important to recognize that technical advances in refractive surgery are relatively new, and several questions still need to be answered. The hypothesis of Motowanii in the Layer Yolked Reduction of Astigmatism (LYRA) protocol [30-32], in which the difference between the cylinders measured on the anterior surface of the cornea and the total astigmatism is mainly due to the 
interference of the HOAs, remains controversial. Other factors, such as a posterior cornea, lens, vitreous, and retina, may be responsible for residual ocular astigmatism (ORA). Recent studies [33, 34] with a new analytics algorithm, the Phorcides Analytic Engine (Phorcides LLC), show promise in attempting to fill this gap. Lobanoff et al. [33] explained in his article that the FDA study for approval of Contoura only included patients demonstrating a close proximity between the clinical cylinder and that measured in the topography and excluded those who had a significant disparity between the measurements; only $24 \%$ of patients initially selected for the study were included, which significantly limited the indication of the technique by the criteria approved by the FDA.

Additionally, according to Lobanoff, in addition to anterior surface astigmatism, the Phorcides system considers corneal irregularities that contribute to high-order aberrations, posterior astigmatism and lenticular astigmatism in its ablation calculations, which may be a more effective approach than techniques guided by topography with modification of clinical refraction; however, although promising, this technology remains relatively recent, and its calculations have not yet been fully clarified. New studies are needed to write the newest chapter on customized laser surgery.

In conclusion, the correction of myopia with or without astigmatism with both the Contoura system and the Custom-Q system showed excellent visual and refractive results, but the evidence did not reveal any clear differences between these two methods after one year of follow-up.

\section{Abbreviations}

FemtoLASIK

femtosecond laser-assisted in situ keratomileusis

TCAT

topography-guided customized ablation

Custom-Q

asphericity-guided ( $Q$ ) customized ablation

UDVA

uncorrected distance visual acuity

CDVA

corrected distance visual acuity

VA

visual acuity

MRSE

manifest refractive spherical equivalent

CYL

cylinder

PACHY

pachymetry

PTA 
percentage of altered tissue

RMS

total corneal aberrations root mean square

DEF

defocus

TRE

trefoil

SA

spherical aberration

\section{Declarations}

\section{Ethics approval and consent to participate}

Informed consent was obtained from all participants and the studies were conducted in accordance with the Declaration of Helsinki.

Approved by the ethics committee of the Hospital Complex HUOC/PROCAPE, RECIFE-PE BRAZIL.

$\mathrm{CAAE}=96778718.9 \cdot 0005192$

$\mathrm{CEP}=2979275$

Consent for publication

Not applicable

\section{Availability of data and materials}

All data are available from OFTALMAX, Recife-PE. Data can be accessed upon request to the authors, represented by the main author.

\section{Competing interests}

No potential competing interests are reported by the authors.

\section{Funding}

This work was supported by Oftalmax, Recife, PE Brazil and Alcon (Fort Worth, Tx, USA). The sponsors had no role in the design or conduct of this research.

\section{Author contributions}

LA and MA reviewed the literature and compiled pre- and post-operative data in Excel tables, in addition to helping with the measurement of UDVA and randomization in the operating room. 
MT,NM, CB and AM performed the complete ophthalmologic exams before and after surgery. They also helped in writing the manuscript.

AFL was responsible for the randomization of patients in the operating room in addition to the personalized surgical planning together with an Alcon consultant for each eye. It also helped in the writing of the manuscript.

EMA was the responsible surgeon and the main author of the manuscript

\section{Acknowledgements}

We are grateful to Oftalmax and Alcon for their financial support. We would like to acknowledge Oftalmax employees for the valuable effort and commitment they dedicated to our patients during all phases of the project. We also thank Renato Maraldi and Erika Lima (Alcon consultants) for the dedication and kindness that they gave our entire team and patient cohort during the weekends when we performed the surgeries.

\section{Authors' information}

EMA has been a refractive surgeon for 20 years and all authors involved have a deep interest in the subject and, like AFL, he is pursuing a doctorate in ophthalmology at Escola Paulista de Medicina, UNIFESP. AFL, NM and CB are also refractive surgeons at another eye center. Our motivation in this study was to find the best method to deliver a better result to our patients, who benefited from a high-level surgical procedure at no cost. All patients involved were fully satisfied with the final result.

\section{References}

1. Holden BA, Fricke TR, Wilson DA, Jong M, Naidoo KS, Sankaridurg P, et al. Global prevalence of myopia and high myopia and temporal trends from 2000 through 2050. Ophthalmology. 2016;123:1036-42.

2. Sandoval HP, Donnenfeld ED, Kohnen T, Lindstrom RL, Potvin R, Tremblay DM, et al. Modern laser in situ keratomileusis outcomes. J Cataract Refract Surg. 2016;42:1224-34.

3. Kim TI, del Barrio JLA, Wilkins M, Cochener B, Ang M. Refractive surgery. Lancet 2019;393:2085-98.

4. Farooqui MA, Al-Muammar AR. Topography-guided CATz versus conventional LASIK for myopia with the NIDEK EC-5000: a bilateral eye study. J Refract Surg. 2006;22:741-5.

5. El Awady HE, Ghanem AA, Saleh SM. Wavefront-optimized ablation versus topography-guided customized ablation in myopic LASIK: comparative study of higher order aberrations. Ophthalmic Surg Lasers Imaging. 2011;42:314-20.

6. Jain AK, Malhotra C, Pasari A, Kumar P, Moshirfar M. Outcomes of topography-guided versus wavefront-optimized laser in situ keratomileusis for myopia in virgin eyes. J Cataract Refract Surg. 2016;42:1302-11. 
7. Tawfik A, Eid AM, Hasanen R, Moftah IA. Q-value customized ablation (custom-Q) versus wavefront optimized ablation for primary myopia and myopic astigmatism. Int Ophthalmol. 2014;34:259-62.

8. Koller T, Iseli HP, Hafezi F, Mrochen M, Seiler T. Q-factor customized ablation profile for the correction of myopic astigmatism. J Cataract Refract Surg. 2006;32:584-9.

9. Zhang Z, Wang J, Niu W, Ma M, Jiang K, Zhu P, et al. Corneal asphericity and its related factors in 1052 Chinese subjects. Optom Vis Sci. 2011;88:1232-9.

10. Sinjab MM, Cummings AB. Customized laser vision correction. Springer; 2018.

11. Nattis AS, Rosenberg ED, Mcdonald MB, Donnenfeld ED. Topography-guided ablations: early us experience and utility across the refractive landscape. Curr Ophthalmol Rep. 2017;5:232-8.

12. Kanellopoulos AJ. Topography-guided custom retreatments in 27 symptomatic eyes. J Refract Surg. 2005;21:S513-8.

13. Lin DT, Holland SR, Rocha KM, Krueger RR. Method for optimizing topography-guided ablation of highly aberrated eyes with the ALLEGRETTO WAVE excimer laser. J Refract Surg. 2008;24:S439-45.

14. Shetty R, D'Souza S, Srivastava S, Ashwini R. Topography-guided custom ablation treatment for treatment of keratoconus. Indian J Ophthalmol. 2013;61:445-50.

15. Kymionis GD, Portaliou DM, Kounis GA, Limnopoulou AN, Kontadakis GA, Grentzelos MA. Simultaneous topography-guided photorefractive keratectomy followed by corneal collagen crosslinking for keratoconus. Am J Ophthalmol. 2011;152:748-55.

16. U.S. Food and Drug Administration. Summary of safety and effectiveness data [for the ALLEGRETTO wave eye-Q excimer laser]. 2003. https://www.accessdata.fda.gov/cdrh_docs/pdf2/P020050S012b.pdf. Accessed 27-Sep 2013

17. Santhiago MR, Smajda D, Wilson SE, Randleman JB. Relative contribution of flap thickness and ablation depth to the percentage of tissue altered in ectasia after laser in situ keratomileusis. J Cataract Refract Surg. 2015;41:2493-500.

18. Santhiago MR, Giacomin NT, Smadja D, Bechara SJ. Ectasia risk factors in refractive surgery. Clin Ophthalmol. 2016;10:713-20.

19. Stojanovic A, Wang L, Jankov MR, Nitter TA, Wang Q. Wavefront optimized versus custom-Q treatments in surface ablation for myopic astigmatism with the wavelight ALLEGRETTO laser. J Refract Surg. 2008;24:779-89.

20. Alpins N, Stamatelatos G. Customized photoastigmatic refractive keratectomy using combined topographic and refractive data for myopia and astigmatism in eyes with forme fruste and mild keratoconus. J Cataract Refract Surg. 2007;33:591-602.

21. Kanellopoulos AJ. Topography-modified refraction (TMR): adjustment of treated cylinder amount and axis to the topography versus standard clinical refraction in myopic topography-guided LASIK. Clin Ophthalmol. 2016;10:2213-21.

22. De Stefano VS, Meister C, Ehlke GL, Krueger RR. Analysis of planning strategies in primary eyes gaining a line or more of visual acuity after topography-guided laser in situ keratomileusis. J 
Cataract Refract Surg. 2019;45:321-7.

23. Shetty R, Shroff R, Deshpande K, Gowda R, Lahane S, Jayadev C. A prospective study to compare visual outcomes between wavefront-optimized and topography-guided ablation profiles in contralateral eyes with myopia. J Refract Surg. 2017;33:6-10.

24. Tiwari NN, Sachdev GS, Ramamurthy S, Dandapani R. Comparative analysis of visual outcomes and ocular aberrations following wavefront optimized and topography-guided customized femtosecond laser in situ keratomileusis for myopia and myopic astigmatism: A contralateral eye study. Indian J Ophthalmol. 2018;66:1558-61.

25. Kim J, Choi SH, Lim DH, Yang CM, Yoon GJ, Chung TY. Topography-guided versus wavefrontoptimized laser in situ keratomileusis for myopia: surgical outcomes. J Cataract Refract Surg. 2019;45:959-65.

26. Wallerstein A, Gauvin M, Qi SR, Bashour M, Cohen M. Primary topography-guided LASIK: treating manifest refractive astigmatism versus topography-measured anterior corneal astigmatism. J Refract Surg. 2019;35:15-23.

27. Ozulken K, Yuksel E, Tekin K, Kiziltoprak H, Aydogan S. Comparison of wavefront-optimized ablation and topography-guided contoura ablation with LYRA protocol in LASIK. J Refract Surg. 2019;35:2229.

28. Zhang Y, Chen Y. Topography-guided versus wavefront-optimized FS-LASIK for correcting myopia and myopic astigmatism. J Refract Surg. 2019;35(9):575-582.

29. Skanchy DF, Linn SH, Kang P, Durrie DS. Comparison and analysis of FDA reported visual outcomes of the three latest platforms for LASIK: wavefront guided Visx iDesign, topography guided WaveLight Allegro Contoura, and topography guided Nidek EC-5000 CATz. Clin Ophthalmol 2017;11:135-147

30. Motwani M. The use of wavelight(®) contoura to create a uniform cornea: The lyra protocol. Part 1: the effect of higher-order corneal aberrations on refractive astigmatism. Clin Ophthalmol. 2017;11:897-905.

31. Motwani M. The use of wavelight(B) contoura to create a uniform cornea: the LYRA protocol. Part 2: the consequences of treating astigmatism on an incorrect axis via excimer laser. Clin Ophthalmol. 2017;11:907-13.

32. Motwani M. the use of wavelight(B) contoura to create a uniform cornea: the LYRA protocol. Part 3: The results of 50 treated eyes. Clin Ophthalmol. 2017;11:915-21.

33. Lobanoff M, Stonecipher K, Tooma T, Wexler S, Potvin R. Clinical outcomes after topography-guided LASIK: comparing results based on a new topography analysis algorithm with those based on manifest refraction. J Cataract Refract Surg. 2020;46:814-9.

34. Stulting RD, Durrie DS, Potvin RJ, Linn SH, Krueger RR, Lobanoff MC, et al. Topography-guided refractive astigmatism outcomes: predictions comparing three different programming methods. Clin Ophthalmol. 2020;14:1091-100.

\section{Figures}




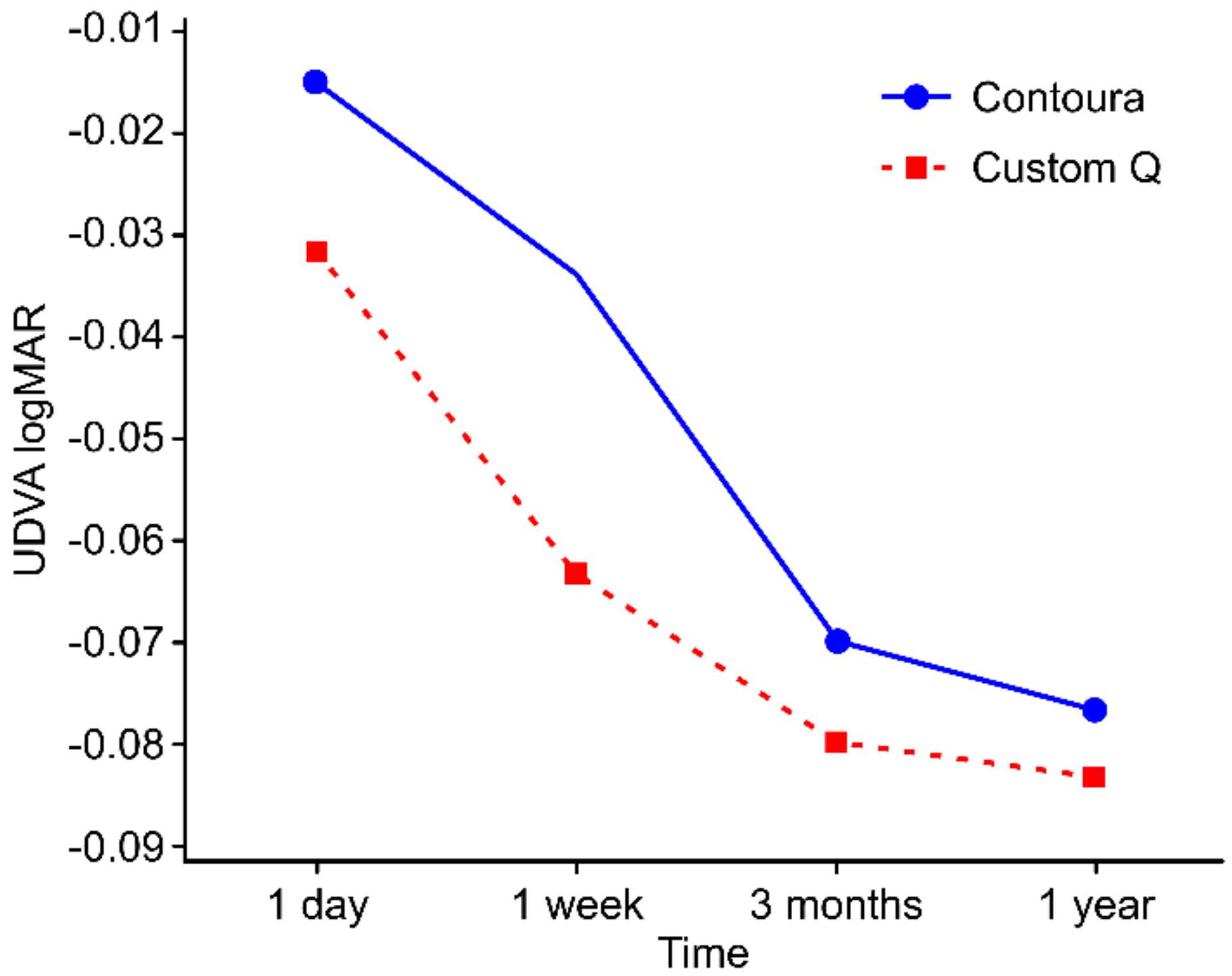

Figure 1

Uncorrected distance visual acuity assessment (UDVA) (mean). Embedded text: Interaction Graph for UDVA UDVA (mean logMAR) Surgery Contoura/Custom-Q Day 1/Week 1/3 months/ 1 year Assessment 
A

A 30 eyes (plano target)

11 months postop

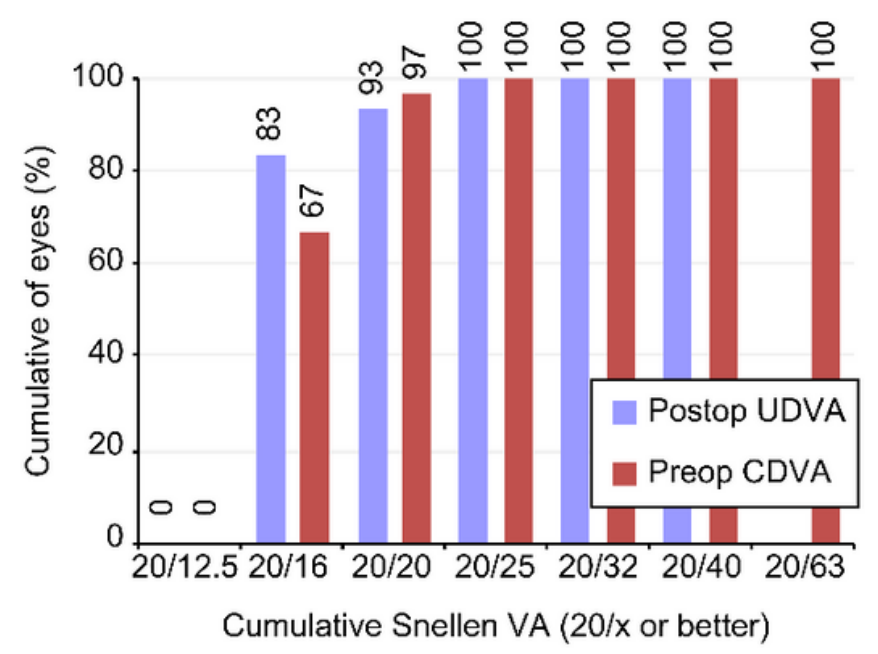

C

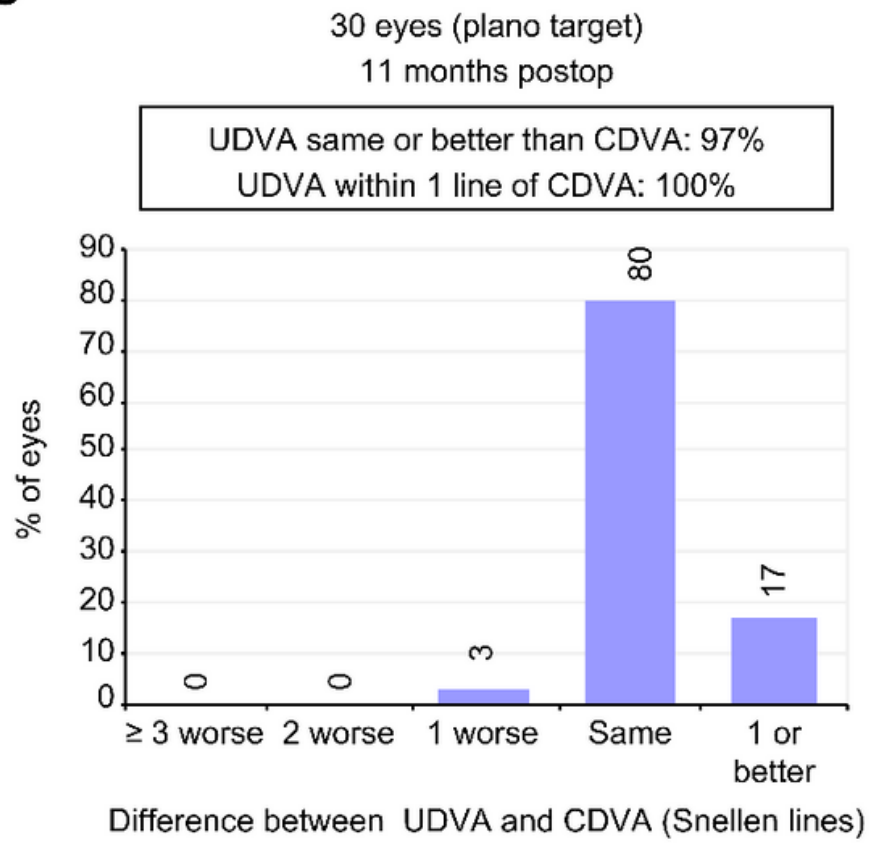

B

30 eyes (plano target)

12 months postop

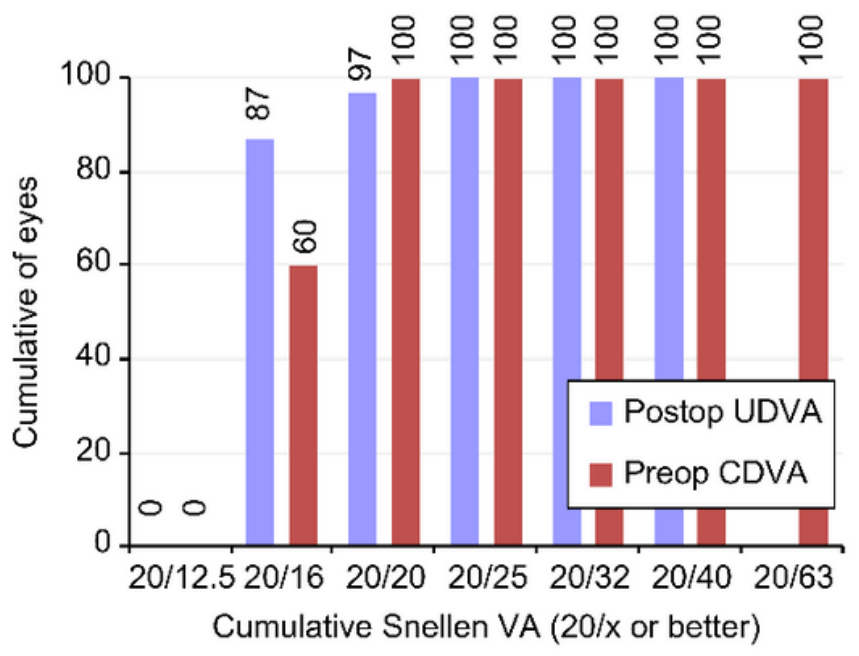

D

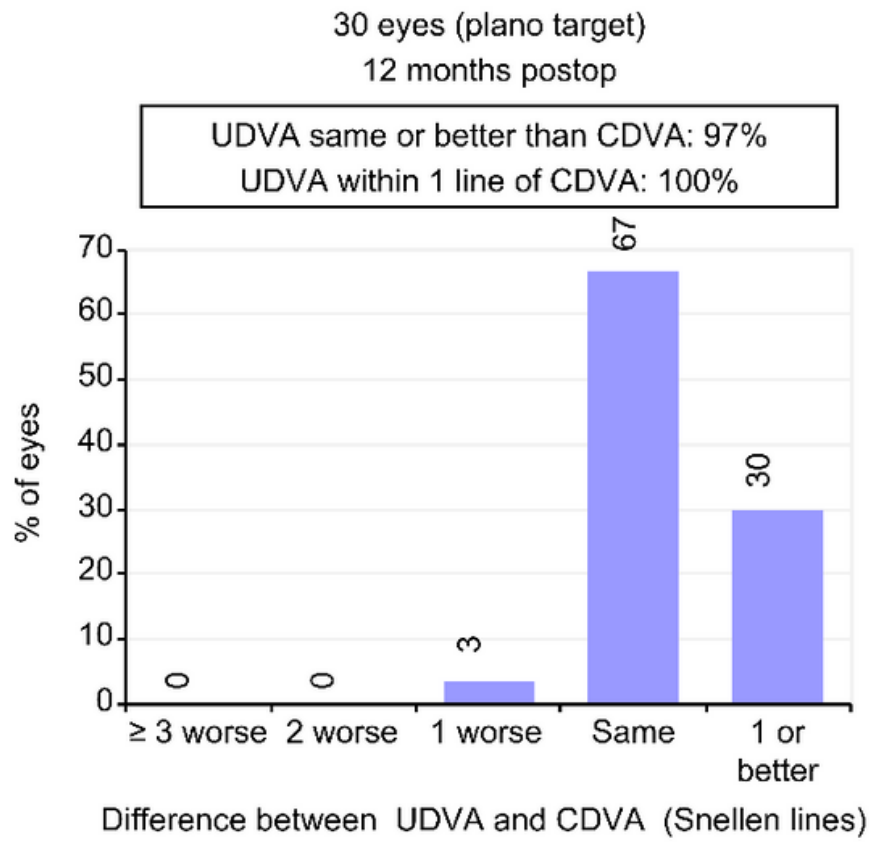

Figure 2

Refractive and visual outcomes, $\mathrm{A}$ and $\mathrm{C}=$ Contoura, $\mathrm{B}$ and $\mathrm{D}=$ Custom- $\mathrm{Q}$ (UDVA=uncorrected distance visual acuity; CDVA= corrected distance visual acuity; $\mathrm{VA}=$ visual acuity). 
A

30 eyes

11 months postop

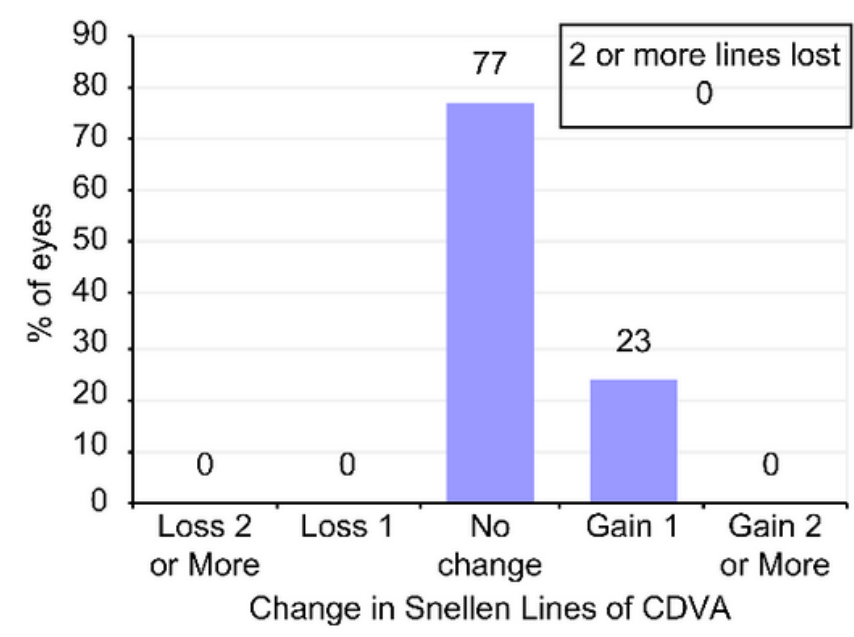

C

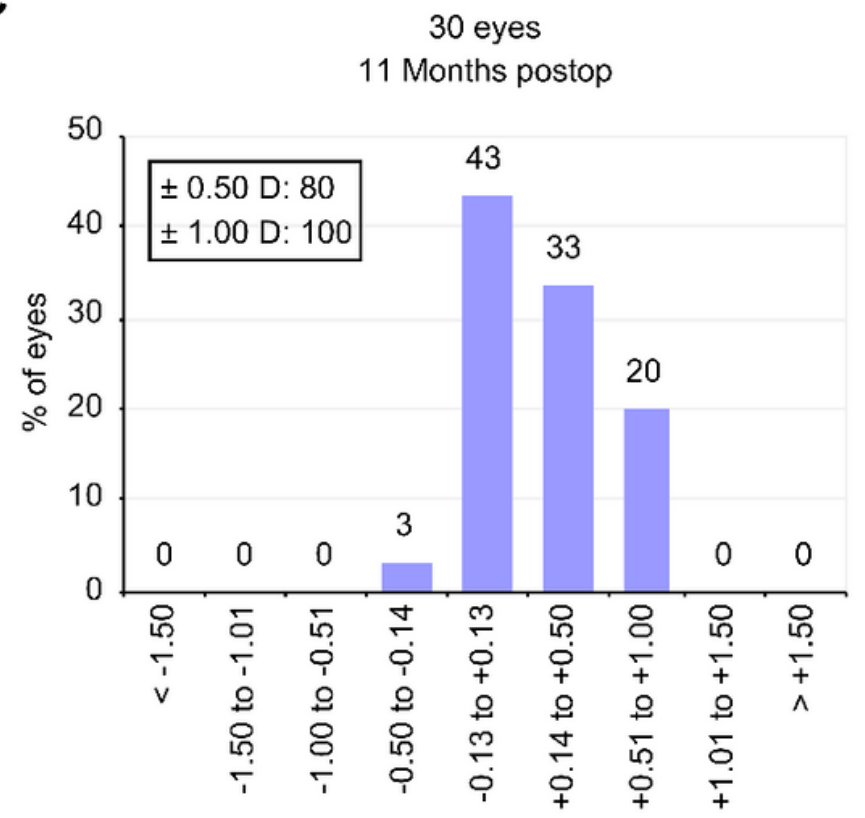

Accuracy of SEQ to intended target (D)
B

30 eyes

12 months postop

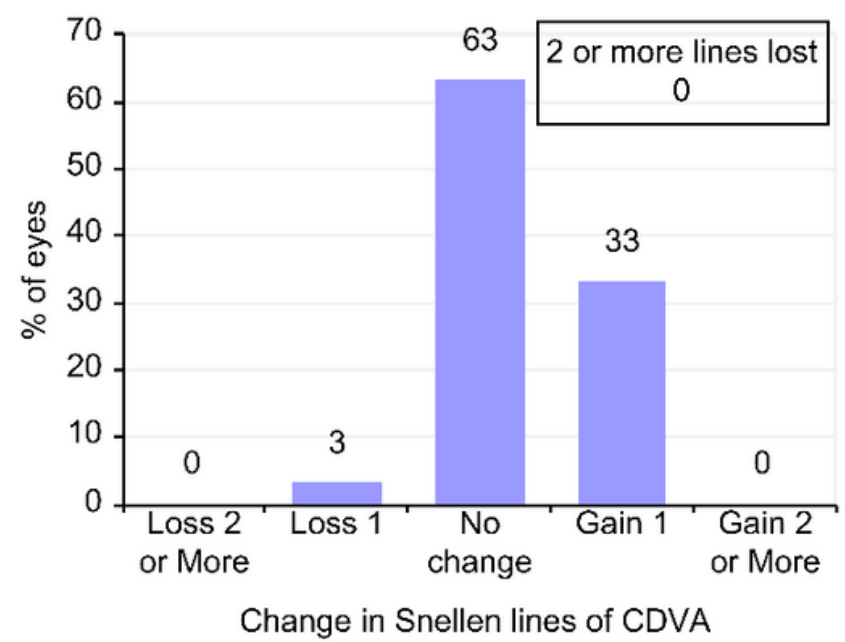

D

30 eyes

12 months postop

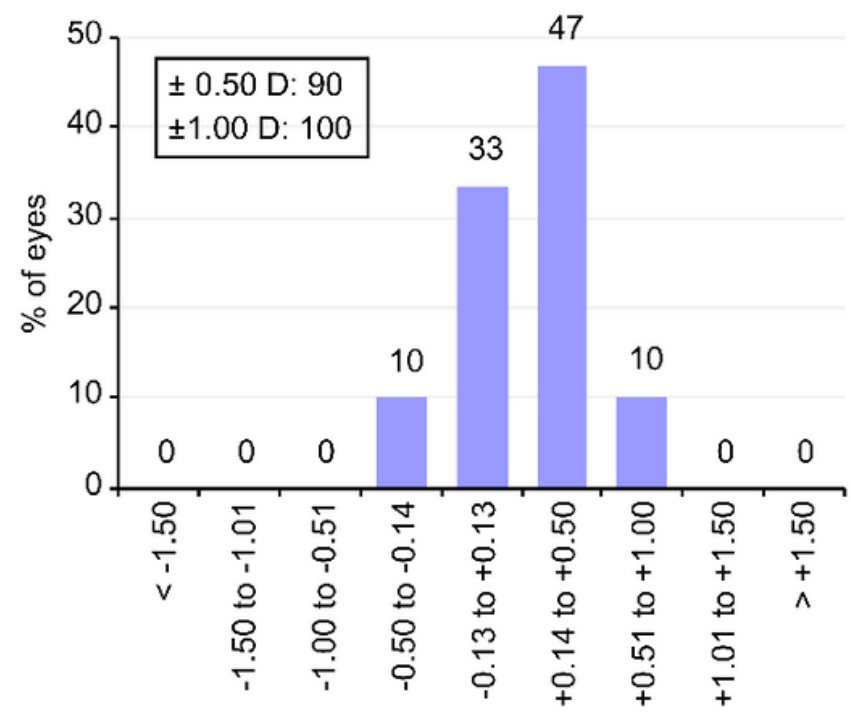

Accuracy of SEQ to intended target (D)

\section{Figure 3}

Refractive and visual outcomes, $\mathrm{A}$ and $\mathrm{C}=$ Contoura, $\mathrm{B}$ and $\mathrm{D}=$ Custom- $\mathrm{Q}$ (CDVA= corrected distance visual acuity, $S E Q=$ spherical equivalent, $D=$ diopter). 
A

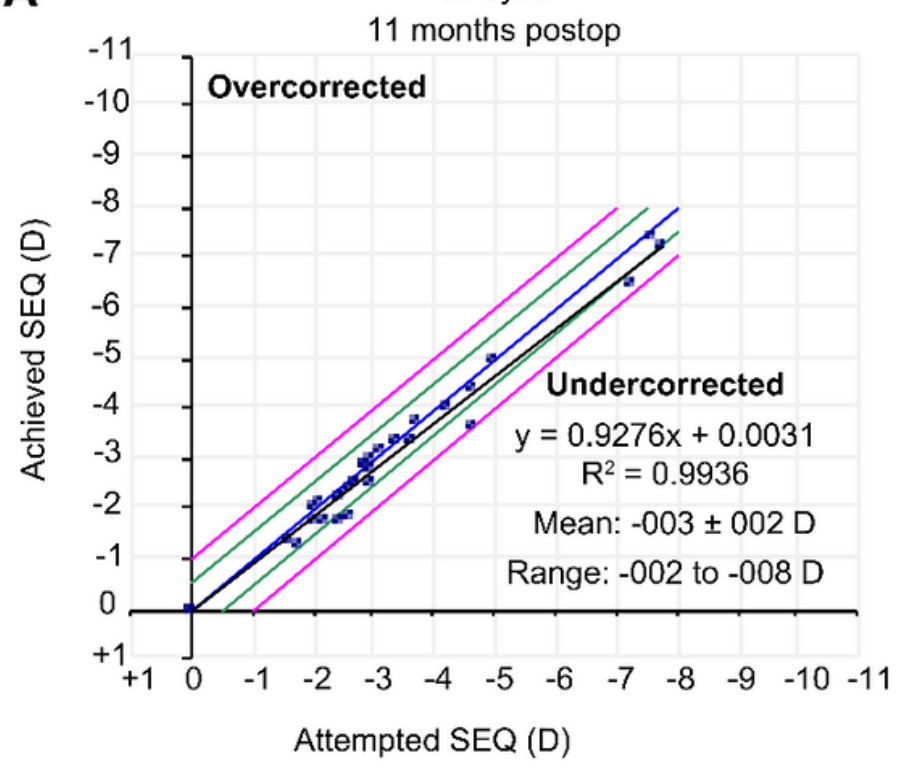

C

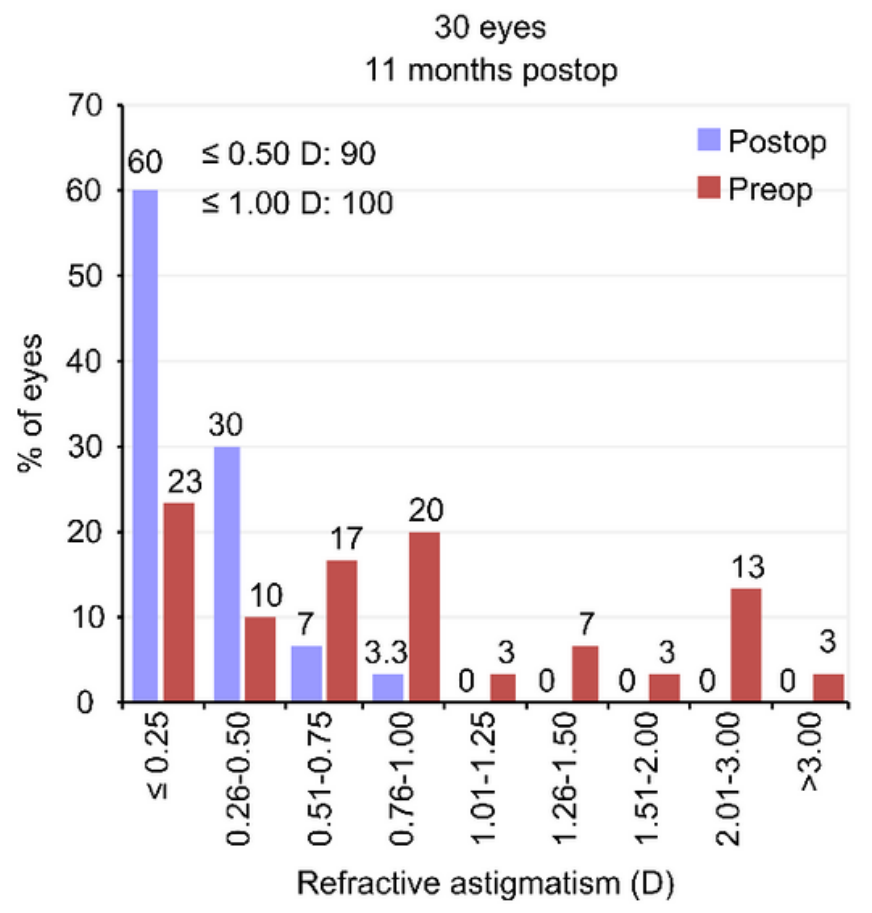

B

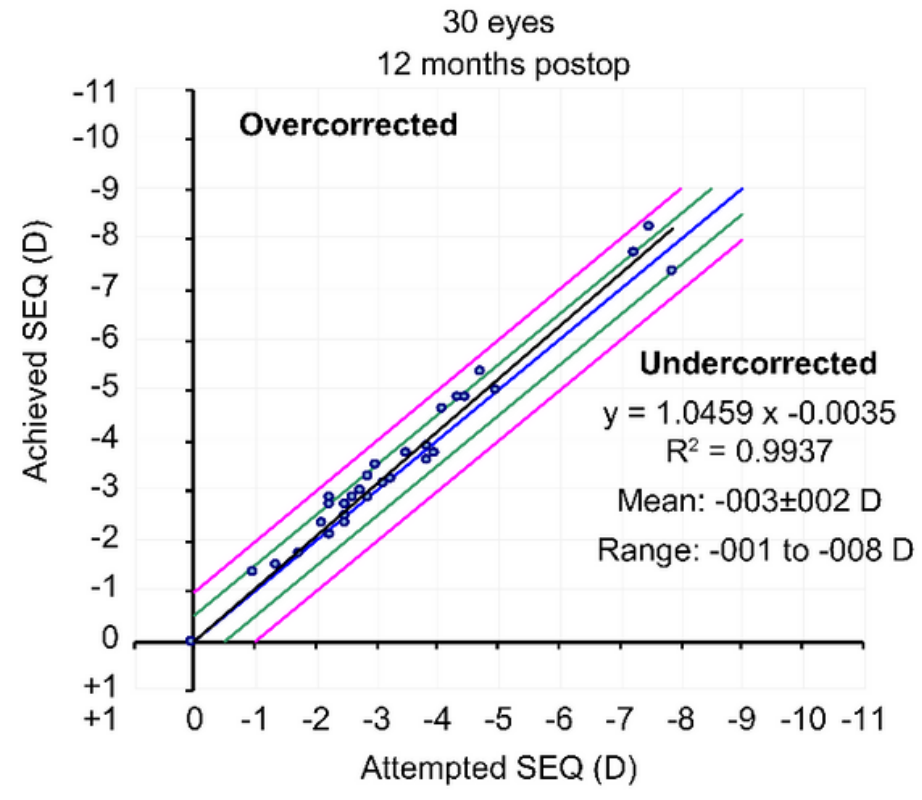

D

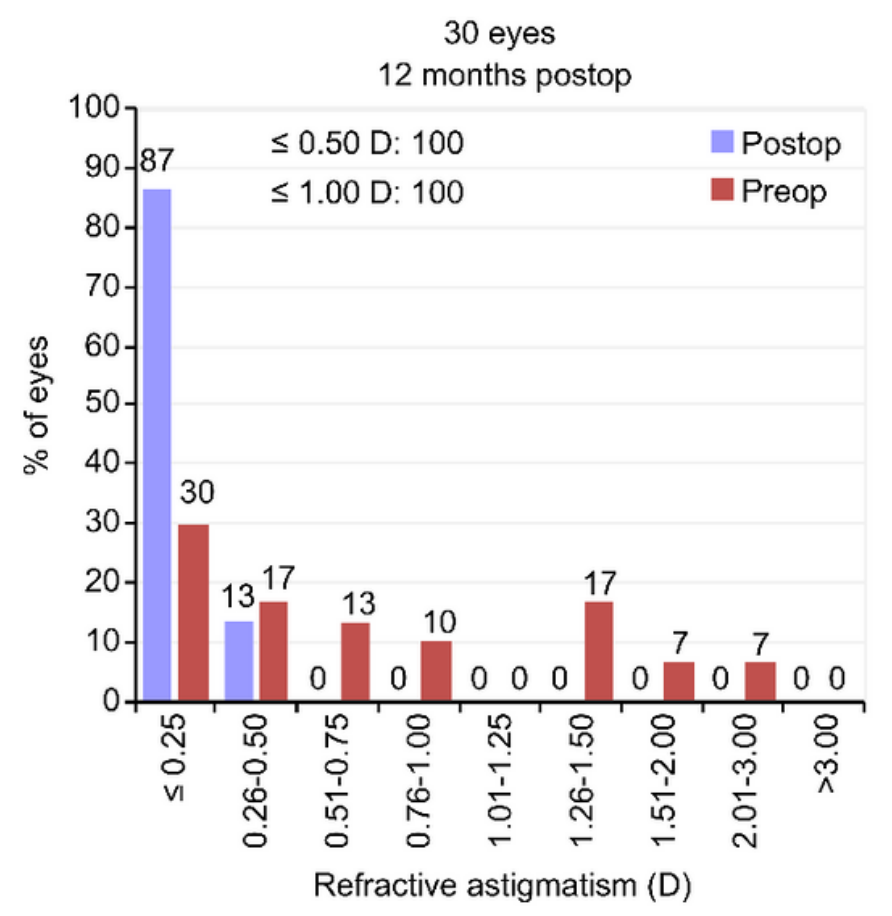

\section{Figure 4}

Refractive and Visual outcomes (CDVA= corrected distance visual acuity, SEQ=spherical equivalent, $\mathrm{D}=$ diopter) 